\title{
Maurice Cosandey, une sorte c'rambassadeur de la chimie
}

\section{Entretien avec le professeur Maurice Cosandey}

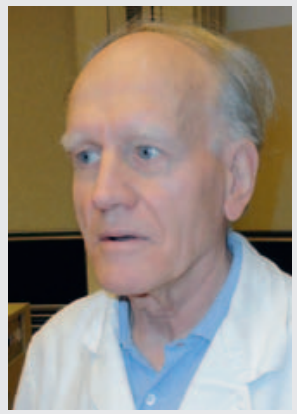

Maurice Cosandey, professeur à la retraite du Gymnase de Chamblandes à Pully-Lausanne, a été récompensé en 2009 par la Médaille de la Société Suisse de Chimie pour sa contribution exceptionnelle à l'enseignement de la chimie. Aujourd'hui encore, il sélectionne et entraîne les gymnasiens prometteurs qui défendent les couleurs de la Suisse aux Olympiades de chimie et il organise des journées de démonstrations de chimie pour des maîtres de gymnase à l'EPFL.

David Spichiger, SCS Executive Director, a eu l'occasion de parler avec M. Cosandey à l'EPFL et de suivre une de ses démonstrations. Il lui a posé quelques questions.

M. Cosandey, aujourd'hui 13 septembre 2011, 82 maîtres de chimie de niveau gymnase sont réunis dans un des grands auditoires de l'EPFL. Quel est le but de cette journée?

Le but de cette journée est de permettre à tous les maîtres de chimie de Romandie de présenter à leurs collègues quelquesunes de leurs démonstrations de cours ou de travaux pratiques, et de discuter de leur valeur, entre nous. Chacun peut s'exprimer librement pour proposer une variante ou une modification.

Quelle est votre motivation pour vous engager à ce point après la retraite?

J'ai hélas dî constater que la plupart des jeunes maîtres qui arrivent dans l'enseignement au terme de leurs études sont bien formés théoriquement, mais qu'ils ont souvent des lacunes énormes au niveau pratique, et qu'ils en souffrent. Résultat: ils préfèrent enseigner avec une craie et un tableau noir, plutôt que de faire des démonstrations pratiques. J'ai même constaté qu'ils avaient parfois peur de se lancer dans des expériences. La peur est mauvaise conseillère. J'ai décidé de faire un effort en leur faveur et de montrer comment enseigner la chimie réelle avec des éprouvettes.

Comment et quand tout cela a commencé?

Il existe une petite société très vivante dans le canton de Vaud, dite MAGYC, acronyme de Maîtres de gymnase en chimie. Nous avons environ 4 séances par année, et parlons en général de problèmes administratifs. Un jour, j'ai proposé de présenter une démonstration en entrée de séance à chaque réunion du MAGYC. L'idée a plu. Et comme mon initiative a eu du succés, d'autres maîtres vaudois ont suivi, et ont décidé spontanément de présenter leur démonstration à la MAGYC, le tout sans s'annoncer au préalable.

En 2007, vu l'enthousiasme des collègues, j'ai proposé de consacrer une journée complète à ces démonstrations et d'étendre l'audience à toute la Romandie. J'ai demandé à 4 ou 5 collègues vaudois de présenter une ou plusieurs démonstrations à l'EP$F L$, sans se soucier de la durée de leur intervention. Je prenais l'engagement de remplir le reste du temps avec mes expériences. En 2008, il y a eu 48 participants et nous avons présenté en public peut-être une trentaine d'expériences, dont la moitié de ma part. Vu le succès de l'opération, nous l'avons répété, et il y a eu 60 participants en 2009, 71 en 2010 et 82 en 2011. Nous ne sommes jamais arrivés à accomplir le programme prévu dans le temps imparti, donc de $9 \mathrm{~h}$ à 16 h. Mais les participants sont tous enchantés et le disent.

Il faut dire que je suis presque un démonstrateur né. Mon père était droguiste, et, quand j'étais petit, il m'entraînait dans l'arrière-boutique en fin de journée. Rassurez-vous. Je n'ai jamais eu d'accident.

Pensez-vous avoir déjà atteint le plafond avec ce projet ou quels sont les plans pour l'avenir? Est-ce qu'il est prévu d'élargir le projet à la Suisse alémanique?

Nous allons continuer en septembre 2012, à la même date, donc une semaine avant la rentrée universitaire. Cette date convient très bien, parce que les auditoires sont encore libres toute une journée. J'ai déjà des maîtres qui se sont annoncés pour présenter une de leurs démonstrations. Rien n'est prévu pour la Suisse alémanique. Mais il faudrait le faire. Il faudrait surtout que quelqu'un lance l'idée, et en convainquent les collègues.

Combien coûte cette journée pour un participant? Est-ce que la journée est acceptée par la commission d'école comme jour de formation permanente?

La journée coûte fr. 10.-. La journée n'est pas considérée comme officielle hélas. Les maîtres doivent demander congé. La Haute Ecole Pédagogique ne soutient cette initiative que très modérément. L'an dernier certains maîtres n'ont pas obtenu congé à la HEP Lausanne. Je me demande parfois s'ils ne seraient pas un peu jaloux du succès de cette journée, qui est organisée sans eux.

Avez-vous d'autres activités en rapport avec la chimie?

Oui. Je vais souvent à l'étranger présenter mes démonstrations, à Barcelone, Besançon et Copenhague rien qu'en 2011.

Je suis aussi le créateur de la participation suisse aux Olympiades internationales de chimie, il y a 24 ans, et j'entraîne toujours l'équipe de Suisse en organisant les examens de sélection et un weekend d'entraînement à l'EPFL.

Je suis encore le rédacteur francophone d'une petite revue bilingue destinée aux maîtres de chimie suisses, qui s'appelle $C+B$ (pour Chimie + Biologie).

D'autre part, l'EPFL m'engage pour enregistrer des DVD de démonstrations de chimie. Le troisième est terminé et je vais commencer le quatrième en janvier 2012.

Et enfin, en cette année Internationale de chimie 2011, j'anime un stand de démonstrations de chimie en public au centre d'achats Littoral Centre, à Allaman, à la sortie des caisses de la COOP.

Merci beaucoup pour votre temps et encore bonne chance pour tous les projets prévus. 